\title{
Telomere length dynamics in the human pituitary gland: robust preservation throughout adult life to centenarian age
}

\author{
Naoshi Ishikawa • Ken-Ichi Nakamura • Naotaka Izumiyama • Junko Aida • \\ Motoji Sawabe • Tomio Arai • Hiroshi Kishimoto • Mutsunori Fujiwara • \\ Akio Ishii • Kaiyo Takubo
}

Received: 29 September 2010 / Accepted: 14 June 2011 /Published online: 7 July 2011

(C) The Author(s) 2011. This article is published with open access at Springerlink.com

\begin{abstract}
Many studies have demonstrated the association between telomere length in mitotic cells and aging, but the relationship between telomere length in post-mitotic cells and aging in human populations has remained largely unclear. In this study, we assessed the dynamics of telomere length (terminal restriction fragment length-TRF) in normal pituitary glands obtained at autopsy from 65 patients aged between 0
\end{abstract}

Electronic supplementary material The online version of this article (doi:10.1007/s11357-011-9280-y) contains

supplementary material, which is available to authorized users.

N. Ishikawa $(\bowtie) \cdot$ K.-I. Nakamura $\cdot N$. Izumiyama

J. Aida $\cdot$ M. Sawabe $\cdot$ T. Arai $\cdot$ A. Ishii $\cdot$ K. Takubo

Research Team for Geriatric Pathology,

Tokyo Metropolitan Institute of Gerontology,

Sakaecho 35-2 Itabashi-ku,

Tokyo 173-0015, Japan

e-mail: naoshi@tmig.or.jp

H. Kishimoto

Department of Pathology,

Saitama Children's Medical Center,

Iwatsuki-shi,

Saitama-ken 339-8551, Japan

M. Sawabe $\cdot$ T. Arai

Department of Pathology,

Tokyo Metropolitan Geriatric Hospital,

Tokyo 173-0015, Japan

M. Fujiwara

Department of Pathology,

Japanese Red Cross Medical Center,

Tokyo 150-8935, Japan and 100 years. The initial length (the value for neonatal pituitary) was $14.2 \pm 1.1 \mathrm{kbp}$ (mean of the median $\mathrm{TRF}$ value $\pm \mathrm{SD}$ ), being the longest among those for other systemic organs. The annual telomere reduction rates for pituitary $(21 \mathrm{bp})$ were equivalent to or below the lowest values for various cells and tissues. Hence, comparison of TRF lengths among organs revealed that the pituitary gland retained longer telomeres than most other organs throughout life. The median TRF value showed a significant decrease between newborns and the 61-75 age group, but then leveled off or increased slightly in advanced age groups. These data indicate that innate telomere length is highly conserved in the pituitary and suggest that pituitary telomere length provides a good basic indicator for studies of telomere biology.

Keywords Telomere - Terminal restriction fragment (TRF) · Pituitary gland $\cdot$ Human aging $\cdot$ Cell proliferation

\section{Introduction}

Telomeric DNA protects chromosomes against degeneration, reconstruction, fusion, and loss (Blackburn 1991). With the exception of immortal, carcinomatous, and germ cells, the telomeres of all human cells become shortened at every cell division due to the end-replication problem (Olovnikov 1971, 1996; Watson 1972; Levy et al. 1992) and trimming by 
nucleases (Verdun and Karlseder 2007). Indeed, accumulated data indicate that telomeric repeats of DNA sequences at chromosome ends are shortened by $33-120 \mathrm{bp}$ with each cell division in human fibroblasts (Harley et al. 1990) and lymphocytes (Vaziri et al. 1993) in vitro, and that telomeres in most human systemic organs/tissues also shorten by 25-100 bp annually in vivo (Takubo et al. 2002; Ishii et al. 2006). In the case of post-mitotic cells, the situation is quite different. When we analyzed a typical organ composed of post-mitotic cells, the cerebrum, we found that innate telomere lengths were very well maintained and associated with longevity (Nakamura et al. 2007).

The telomere hypothesis of cellular aging suggests that when telomere shortening (on a particular chromosome) reaches a critical level, the chromosome may become unstable and the cells stop dividing (Harley et al. 1990; Allsopp et al. 1992). This hypothesis has prevailed as one of the most feasible for explaining not only the cause of cellular aging but also aging of individuals. Indeed, Cawthon et al. found that individuals with shorter telomeres of blood had a high mortality rate due to heart disease and infectious disease, thus providing cutting-edge data in this field (Cawthon et al. 2003). We have also reported unequivocal data to support the hypothesis that longer telomeres can protect the genome from instability (a major cause of carcinogenesis) and appear to have a relationship to longevity (Nakamura et al. 2007). Although reports regarding the relationship between telomere length and human aging have accumulated, the dynamics of human telomere status in vivo, especially in post-mitotic cells, remain largely unexplored.

In the present study, we measured the terminal restriction fragment (TRF) length of HinfI- or RsaIdigested genomic DNA from human pituitary glands, and investigated the population dynamics of telomere status and the relationship between telomere length and aging. Since the pituitary gland is one of the human organs that shows the highest degree of preservation during aging (Sawabe et al. 2006) and scarcely shows age-related changes, for example lymphocyte infiltration and fibrosis, it is particularly intriguing from the viewpoint of telomere dynamics.

For measurement of telomere length in most of our previous studies, we have been using the peak values demonstrated on densitometric profiles obtained by
Southern blotting of TRF (Hastie et al. 1990). Although many methods for measurement of telomere length have since been introduced (including PCR and fluorescence in situ hybridization), TRF-based analyses have been considered to represent a gold standard in this field, and have been enhanced by the introduction of many innovative developments in both hardware and software. Recently, we have adopted a software package "Telometric", which shows robust reproducibility, and obtained preliminary data indicating that the peak values obtained using the original method are comparable with the median values calculated using "Telometric". In order to derive confirmatory data for comparison with archived data obtained using different methods, we further tried to assess the relationship between the peak and the median values, and between the mean and the median values of telomere length.

\section{Materials and methods}

Samples from autopsied patients

Pituitary tissue samples from 65 autopsied patients (37 males and 28 females) aged between 0 and 100 years were analyzed by Southern blotting. The patients included nine neonates, four infants $(0.3-$ 0.8 years), one young adult (22 years), one individual in his forties, four in their sixties, 14 in their seventies, 15 in their eighties, 15 in their nineties, and two centenarians (each aged 100 years). Among them, pancreas tissue from 14 patients and heart tissue from four patients were also sampled and analyzed (Supplementary material, Table S1).

Pituitary samples from another set of autopsied patients, comprising 17 males and 13 females aged between 0 and 100 years, were analyzed for the proliferative potential of their component cells by immunostaining for the DNA synthesis phase-specific antigen Ki-67 (Supplementary material, Table S2). The autopsies were performed at Tokyo Metropolitan Geriatric Medical Center, Saitama Children's Medical Center, and the Japanese Red Cross Medical Center. None of the case histories or examination findings suggested pituitary gland-related disease. All samples were stored at $-80^{\circ} \mathrm{C}$ until use. The Tokyo Metropolitan Institute of Gerontology Ethics Committee approved the study protocol. Family members of all 
the autopsied individuals provided signed written consent for use of the materials for scientific research, including DNA analysis.

Histological and immunohistochemical examination

The sampled pituitary glands were examined at autopsy by specialists in anatomical and surgical pathology (M. S., T.A., M.F., H.K., and K.T.), and samples exhibiting autolysis or marked degeneration were excluded.

To assess the proliferation status of the component cells of the pituitary gland, we performed immunostaining of age-matched pituitary gland samples with anti-Ki-67 antibody (monoclonal antibody-MIB-1; Dako Cytomation, Glostrup, Denmark) by the Polymer-Immuno Complex method [ENVISION+/ HRP (DAB); Dako Japan]. Antibody-stained sections were examined quantitatively by two pathologists (N. I., J.A.) who estimated the numbers (\%) of cells showing positive staining.

\section{Preparation of genomic DNA}

Genomic DNA was prepared by the standard method. Briefly, the samples were incubated with $2 \mu \mathrm{g} / \mathrm{ml}$ proteinase $\mathrm{K}$ and $0.8 \%$ sodium dodecyl sulfate (SDS) in lysis buffer $(10 \mathrm{mM}$ Tris- $\mathrm{HCl}, 50 \mathrm{mM} \mathrm{NaCl}$, $50 \mathrm{mM}$ EDTA, 27\% sucrose, and distilled water), followed by repeated phenol-chloroform extraction. To isolate intact high molecular weight DNA, we performed all the procedures as gently as possible.

The extracted genomic DNA was tested for its quality using a biased sinusoidal field gel electrophoresis method (Schwartz and Cantor 1984) with the Genofield system (ATTO, Tokyo, Japan). All of the DNA samples showed smears of more than $100 \mathrm{kbp}$, and thus qualified for subsequent Southern blot analyses.

\section{Measurement of telomere (TRF) lengths}

The procedures used for Southern blot analysis have been described previously in detail (Takubo et al. 1999; Nakamura et al. 2002). In previous experiments, we had used HinfI, which has been the restriction enzyme employed most universally. In addition, we sometimes used RsaI, which is also widely adopted in this field and is stochastically and empirically known to cut eukaryotic genomes more frequently than HinfI (see the New England Biolabs technical support manual). We expected that RsaIdigested TRF would have a shorter subtelomeric stretch than HinfI-digested TRF, and that this would provide further confirmation. Hence, in this study, we used two restriction enzymes: HinfI and RsaI (Roche Biochemicals, Germany). Briefly, aliquots of DNA were digested with a restriction enzyme, and complete cleavage was confirmed. All of the DNA samples were analyzed using HinfI, and 44 were analyzed using RsaI because of shortage of available DNA samples. We performed electrophoresis repeatedly, two to four times for each sample. DNA fragments were then transferred to nylon membranes (Hybond-N + , Amersham, UK) and subjected to hybridization with a (TTAGGG) 4 probe that had been labeled with $\left[\gamma-{ }^{32} \mathrm{P}\right]$ ATP (Amersham). After drying, the membranes were exposed to Imaging Plates (Fuji Photo Film, Tokyo, Japan), and analyzed with a Mac BAS2500 image analyzer (Fuji Photo Film) using the programs Image Reader (version 1.1, Fuji Photo Film) and Mac Bas (version 2.4, Fuji Photo Film).

We obtained representative values for TRF length using the software package Telometric (Grant et al. 2001), and calculated the median and the mean values for the TRF smear signals. At this stage, two samples were disqualified due to suspected degradation of the DNA and were omitted from further study, leaving a final total of 63 cases that were analyzed. We also determined the position giving the peak intensity from the profile of the TRF signal intensity by our conventional method, as described previously (Hastie et al. 1990; Takubo et al. 1999). The data, and mean values for each sample, are shown in Supplementary material, Table S1.

\section{Statistical analysis}

Statistical analyses were performed with StatView software version 5.0 (SAS Institute Inc.). Differences in mean values were assessed for significance by analysis of variance (one-factor factorial ANOVA) and follow-up post hoc tests (Tukey-Kramer test and Bonferroni/Dunn test) among four age groups: 01 years, 61-75 years, 76-90 years, and $>90$ years. Correlation significances were assessed with Fisher's test. Categorical variables were analyzed by Pearson's chi-squared test. To analyze the relationships between the median values and the peak values of TRF, the 
median values and mean values of TRF, and the relationships of the pituitary to other organs, we applied a multiple regression model.

\section{Results}

Histological and immunohistochemical characteristics of pituitary glands

As contamination with other cell types is always a problem in studies of telomere length in human tissues or organs (Greider 1996; Takubo et al. 1999), as opposed to measurements using cultured cell lines, we checked the histological characteristics of the pituitary samples. We analyzed the pituitary en bloc for neonates and infants, and a lateral portion of the gland for adults.

A survey of age-matched samples indicated that the major part of each sample was composed of the anterior lobe (adenohypophysis) (Lopes et al. 2007). None of the age-matched samples exhibited marked inflammatory cell infiltration, but small patches of mild fibrosis were evident in a very small number of specimens from elderly individuals. These findings and our previous observations (Sawabe et al. 2006) suggested that the cells evaluated for pituitary TRF were endocrine-competent cells. Immunostaining for Ki-67 showed that positive cells were more prevalent $(5-10 \%)$ in fetuses at 20 weeks of gestation, present at rates of $0.2-0.5 \%$ in neonates and less than $0.2 \%$ in infants, and negative in adults and the elderly (Supplementary materials, Table S2, Fig. S1). These data reflected the rapid proliferation of the pituitary towards the end of gestation.

Representative TRF lengths in pituitary glands

Representative TRF images obtained by Southern blot analysis of Hinfl- or RsaI-digested genomic DNA derived from the neonatal pituitary glands are shown in Fig. 1a (lanes 1-6). Due to sample quantity limitation, the number of cases analyzed after $R s a \mathrm{I}$ digestion was reduced to 40 . The median values (determined using Telometric software) for TRF signals derived from Hinfl- and RsaI-digested pituitary ranged from 9.2 to $15.8 \mathrm{kbp}(12.6 \pm 1.5 \mathrm{kbp}$; mean \pm SD) and from 9.2 to $15.3 \mathrm{kbp}(11.9 \pm 1.4 \mathrm{kbp}$; mean $\pm \mathrm{SD}$ ), respectively. The relationship between median values of TRF lengths for Hinfl-digested and $R s a$ I-digested samples was examined by scatter plot analysis, and this demonstrated a robust correlation between the paired values (slope $=0.95, p<0.0001$; Supplementary material, Fig. S2).

Annual rate of reduction of telomere length in pituitary glands

The median value of the Hinfl-digested TRF lengths for all 63 subjects was plotted as a function of age (Fig. 2), and this yielded a regression line indicating that the average annual rate of telomere shortening was $21 \mathrm{bp}(p<0.001)$.

The RsaI-digested samples were also analyzed in the same way. The average annual rate of reduction of the median value was 7.5 bp $(p=0.31)$. (Supplementary material, Fig. S3).

Telomere length differences between age groups

Since the median TRF values for Hinfl-digested sample sets from neonates were greater than those from elderly individuals, we divided the samples into four age groups $(0-1,61-75,76-90$, and $>90$ years) and assessed the data among the groups by ANOVA and post hoc tests (mean values \pm SD are shown in Fig. 3). The values were greatest for the neonate-infant samples, and smallest for the 61-75-year age group. Comparison of neonates-infants with other age groups for Hinfl-digested samples showed that telomere (TRF median) shortening was statistically significant in each case (0-1 vs. $61-75,0-1$ vs. $76-90,0-1$ vs. >90). There were no significant differences between any of the combinations among the three age groups (Fig. 3).

The RsaI-digested samples were also categorized and analyzed in the same way. The mean value for neonates was also the greatest and that for the 61-75year age group was the smallest. The telomere shortening profile for the $R s a$ I digests resembled that for Hinfl digests, but showed no significant differences among any of the groups (Supplementary material, Fig. S4).

Reassessment of telomere length dynamics in the older population

Since these inter-age-group analyses suggested that the median TRF lengths in pituitary glands were 


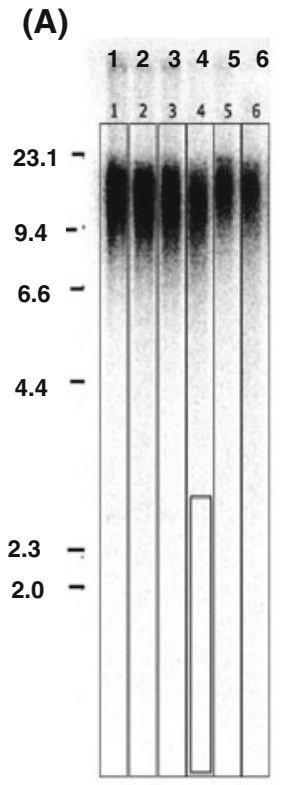

(B)

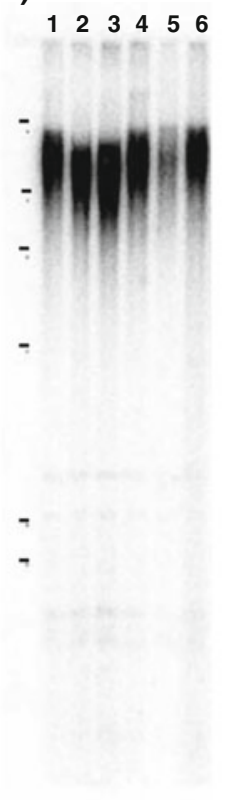

(C)

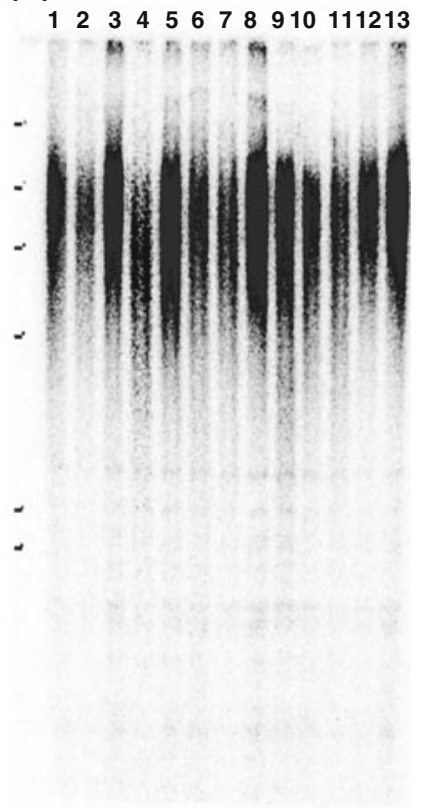

Fig. 1 Southern blot analysis of terminal restriction fragments (TRF) from pituitary and systemic organs. a Representative TRF signals of the pituitary samples obtained from three neonates (case \#8, 38 weeks of gestation-lanes 1 and 2; case\#4, 35 weeks of gestation-lanes 3 and 4; case \#5, 36 weeks of gestationlanes 5 and 6 ). The restriction enzyme employed was either Hinf 1 (lanes 1, 3, 5) or RsaI (lanes 2, 4, 6). The median values (kbp) for the signals were $14.0,13.3,13.4,12.9,14.4$, and 13.7, respectively. b Representative Hinfl-digested TRF signals obtained from a premature neonate (case \#1, 21 weeks of gestation; 1 heart, 2 skin, 3 scalp, 4 adrenal gland, 5 liver, 6 pituitary gland). The median values $(\mathrm{kbp})$ for the signals were

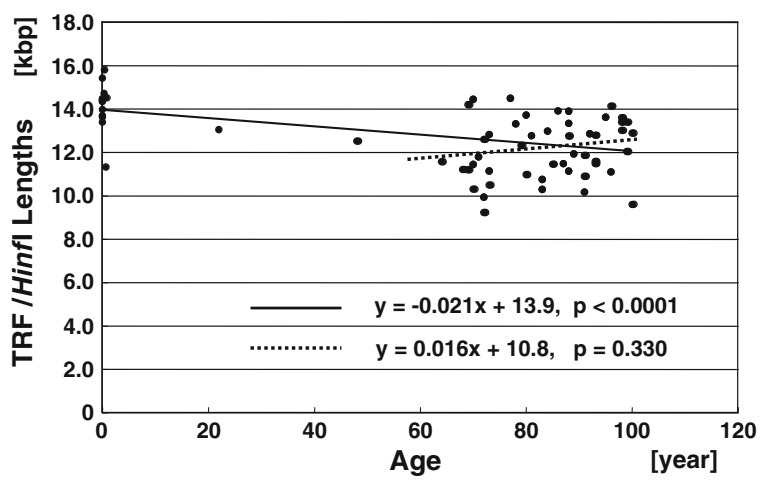

Fig. 2 Telomere dynamics with aging. First scatter plot analysis of representative TRF values obtained from Hinfl-digested samples as a function of donor age over the full range yields a decreasing regression line for each representative [median values; filled circles $(n=63)$, solid line]. Second scatter plot analysis of the TRF values for individuals aged 60 years and older yields an increasing regression line for each representative [median values; filled circles $(n=48)$, dashed line]
$14.8,13.2,13.0,14.6,15.1$, and 15.4, respectively. c Representative Hinfl-digested TRF signals obtained from a centenarian (case \#62; 1 cerebral gray matter, 2 cerebral white matter, 3 heart, 4 liver, 5 kidney, 6 adrenal gland, 7 pancreas, 8 thyroid, 9 tongue, 10 skin, 11 scalp, 12 muscle, 13 pituitary gland). The median values $(\mathrm{kbp})$ for the signals were 8.7, 7.9, 9.2, 6.6, 8.0, 8.1, 7.5, $8.5,7.8,7.4,7.8,8.5$, and 9.1 , respectively. Size marker $(\lambda /$ HindIII) positions are indicated on the left. The mean values (the median values calculated using the Telometric software package, and the peak values of signal intensity) of repeated experiments are summarized in the supplementary table (Supplementary material, Table S1)

preserved in the elderly, we reassessed the scatter plot data for individuals in their sixties and older (omitting neonates, infants, and young adults) (dashed line in Fig. 2). The representative values (median TRF values for Hinfl-digested materials) provided regression lines with positive slope values [18(bp/year)]. The representative values for $R s a$ I-digested materials also provided regression lines with positive slope values (Supplementary material, dashed line in Fig. S3).

Differences in telomere length among the three different methods

To assess the present data against archived data obtained using a different method, we also determined the position giving the peak intensity from the profile of the TRF signal intensity obtained by the conventional method, as described previously (Hastie et al. 


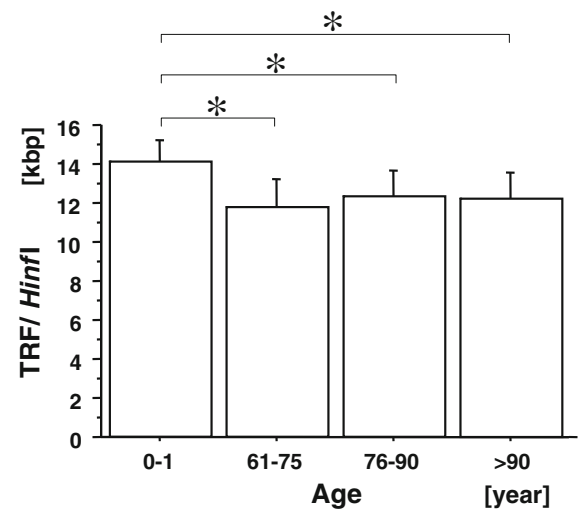

Fig. 3 Comparison of TRF lengths for the pituitary gland in the four age groups: $0-1,61-75,76-90$, and $>90$ years. Mean values $( \pm$ SD) of individual median TRF length for Hinfl-digested samples from the four age groups were calculated and compared using ANOVA and post hoc tests. The mean value $( \pm \mathrm{SD})$ for neonates and infants $(n=13)$ was $14.2 \pm 1.1 \mathrm{kbp}$. The values for the older age groups were $11.6 \pm 1.5 \mathrm{kbp}$ for subjects aged $61-$ 75 years $(n=14), 12.4 \pm 1.3 \mathrm{kbp}$ for those aged $76-90$ years $(n=$ 17 ), and $12.3 \pm 1.3 \mathrm{kbp}$ for those aged $>90$ years $(n=17$, including two centenarians). The $p$ value by ANOVA was $<0.0001$. Each of the differences between the neonates-infants versus the three older age groups was significant by both Tukey-Kramer test and Bonferroni-Dunn test (significant difference, $p<0.0083$, is indicated by asterisks, and $p$ values presented in parentheses were calculated by the latter test): $61-75(p<0.0001), 76-90$ ( $p=$ $0.0007)$, and $>90(p=0.0002)$, respectively. However, there were no significant differences between any of the combinations among the other three age groups

1990; Takubo et al. 1999), and compared it with the data determined from the median values (Supplementary material, Table S1). The median values (determined using the Telometric software package) and the peak values for TRF signals derived from Hinfldigested pituitary ranged from 9.2 to $15.8 \mathrm{kbp}$ and from 8.3 to $18.2 \mathrm{kbp}$, respectively. Scatter plot analysis demonstrated a robust relationship between the median and the peak values (Fig. 4), and the regression lines obtained from the median values and from the peak values showed a close resemblance (Fig. 5). To further investigate the relationship between the median and the peak values, we performed multiple regression analysis. This indicated that the group coefficient $\left(\beta_{2}\right)$ of the median versus peak values was very small $(0.043 \mathrm{kbp})$ and were not statistically significant $(p=0.90)$ (Fig. 5).

We also investigated the relationship between the median and the mean values using the same multiple regression analysis. This demonstrated that the differ- ence between the median versus the mean values was $1.2 \mathrm{kbp}$ and robustly significant $(p<0.0001)$ (Supplementary material, Fig.S5).

Comparison of TRF length between pituitary gland and other organs

We analyzed TRF lengths for both the pituitary gland and pancreas from 14 patients (data are shown in Table S1). TRF length for the pituitary gland was greater (by more than $0.5 \mathrm{kbp}$ ) than that for the pancreas in 10 cases, and the values for the pituitary and pancreas were almost the same (the difference being less than $0.4 \mathrm{kbp}$ ) in four cases (including one premature neonate and one 0.3 -year infant). We also analyzed TRF lengths for both the pituitary gland and heart in four individuals (data are shown in Table S1). TRF length for the pituitary was greater (by more than $0.5 \mathrm{kbp}$ ) than that for the heart in two cases, and the values were almost the same (the difference being less than $0.4 \mathrm{kbp}$ ) in two cases.

In two individuals, we then analyzed the TRF lengths for systemic organs in addition to the pituitary. Representative TRF images of Hinfl-digested genomic DNA derived from the systemic organs of an individual infant are shown in Fig. 1b (lanes 1-6). Median TRF values were within the range 13.0-15.4 kbp, and the pituitary gland had the largest median value. Other representative TRF images derived from systemic organs of a centenarian individual are shown in

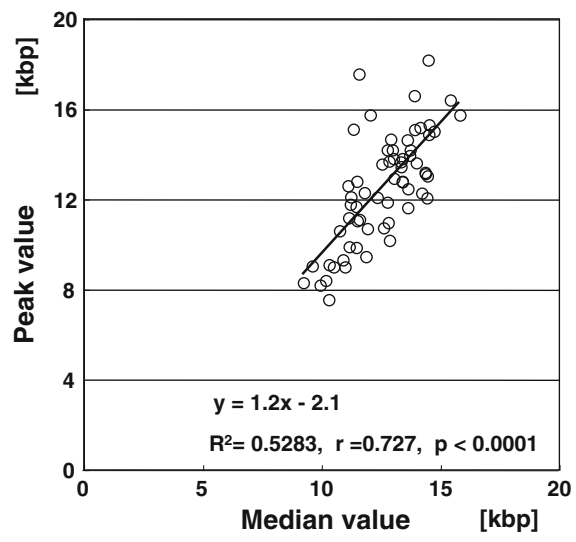

Fig. 4 Relationship between the peak values of TRF signal intensity and the median values calculated using the Telometric software package. Scatter plot analysis of TRF lengths for Hinfl-digested samples provides a robust correlation between the peak values ( $y$-axis) and the median values ( $x$-axis), yielding a regression line 


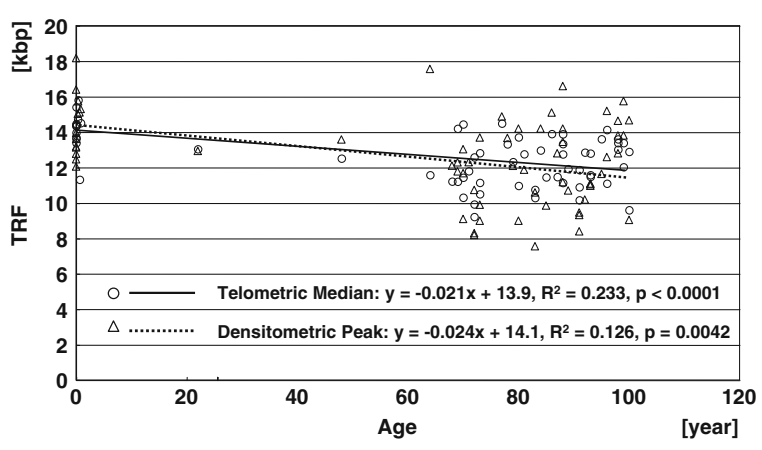

Fig. 5 Comparison between two scatter plot analyses: one regression line was obtained from the median values of TRF as a function of donor age (see Fig. 2), and the other was obtained from the peak values of TRF as a function of donor age. To analyze statistically the relationship between the median values and the peak values of pituitary TRF, we applied a multiple regression model that related $Y$ (TRF length) to $X_{1}$ (age) and $X_{2}$ (group), given that the value for the median group was 1 and that for the peak group was $0: Y=\beta_{0}+\beta_{1} X_{1}$ (age) $+\beta_{2} X_{2}$ (group). The group coefficient $\left(\beta_{2}\right)$ of the median versus peak values was $-0.043 \mathrm{kbp}(p=0.90)$

Fig. 1c (lanes 1-13). Median TRF values for individual organs were within the range $6.6-11.3 \mathrm{kbp}$, and-after the heart - the pituitary gland had the second largest value. These data strongly suggested that the TRF value for the pituitary might be the highest among the systemic organs examined.

We then compared the data derived from pituitary samples with our previous data for in vivo telomere attrition in systemic organs, including the liver, kidney, spleen, brain, heart (Takubo et al. 2002), pancreas (Ishii et al. 2006), and gray matter and white matter of the cerebrum (Nakamura et al. 2007) (shown in Supplementary material, Fig.S7). These comparisons demonstrated that both the regression lines of the TRF values (the median and peak values) for the pituitary gland were higher than those for other organs at all ages between neonates and centenarians except that for gray matter of the cerebrum in individuals over 90 years old. To further investigate the relationship between the TRF values for the pituitary and those of other organs, we performed multiple regression analysis (see the legend for Fig.S7). The probabilities of differences determined by this analysis were as follows: pituitary versus cerebral gray matter, $p=0.0062$; pituitary versus cerebral white matter, $p<0.001$; and pituitary versus heart, $p<0.0001$. The group coefficient $\left(\beta_{2}\right)$ for pituitary versus cerebral gray matter was $0.39 \mathrm{kbp}$ $(p=0.28)$, that for pituitary versus cerebral white matter was $1.45 \mathrm{kbp}(p<0.01)$, and that for pituitary versus heart was $0.82 \mathrm{kbp}(p=0.030)$. The regression line of cerebral cortex located center of regression lines of cerebral gray and white matter, and under of that of heart. These data demonstrated that the TRF for the pituitary was statistically longest among the TRF values for the systemic organs examined except cerebral gray matter.

Comparison of cancer death rates between older age group

The studied individuals were divided into two groups according to the major cause(s) of death diagnosed at autopsy: patients who had died as a result of carcinomas and hematopoietic malignancies, and patients who died of other diseases (see Supplementary material, Table S1, Fig. S6). The latter category included curatively treated cancer patients and patients with latent cancers who had died of other diseases. The cancer death rates for individuals in the 61-75-, 76-90-, and 90-100-year age groups were $79 \%$ (11 cancer deaths among 14 cases), $35 \%(6 / 17)$, and $12 \%(2 / 17)$, respectively (Fig. 6). Statistical analysis revealed a significantly lower rate of cancer-related death in individuals aged 76-90 $(p=0.016)$ and $91-100(p=0.0002)$ years versus those aged $61-75$ years. The malignancies that were considered to have been the direct cause of death were, in order of incidence: lung cancer (5), gastric cancer (3), gallbladder cancer (2), esophageal cancer (2), pancreatic cancer (2), leukemia (acute myelogenous leukemia) (2), colon cancer (1), hepatocellular carcinoma (1), and duodenal cancer (1). The proportion of those who died of cancer was reasonably consistent with that for the Japanese population as a whole (Statistics and Information Department, Ministry's Secretariat, Ministry of Health, Labour and Welfare Japan 2006). For the studied individuals overall, the mean of the median value of TRF length for those who had died of cancer $(n=19-12.2 \pm 1.5 \mathrm{kbp})$ was almost the same as that for those who had died of other diseases $(n=30-12.2 \pm 1.4 \mathrm{kbp})$.

\section{Discussion}

To date, more than 30 human systemic organs or tissues have been subjected to telomere length analysis using various methods. Although many groups have con- 


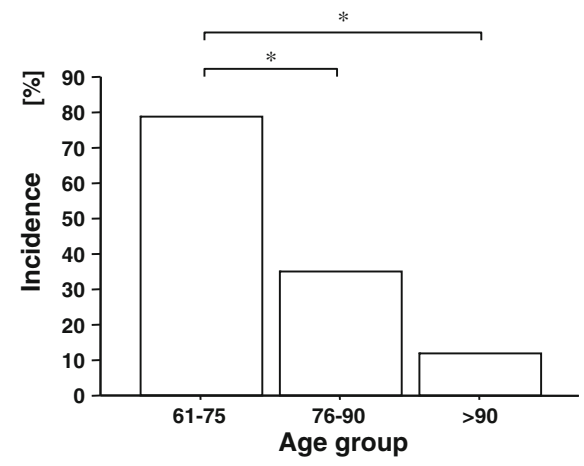

Fig. 6 Cancer death rate in older Japanese age groups. The older patients were divided into three age groups (61-75, 7690 , and $>90$ years). The cancer death rate was $79 \%$ for subjects aged $61-75$ years $(n=11 / 14), 35 \%$ for those aged $76-90$ years $(n=6 / 17)$, and $12 \%$ for those aged $>90$ years $(n=2 / 17$, including two centenarians). Each of the differences between the 61-75-year group versus the two older age groups was significant by Pearson's chi-squared test (significant difference, $p<0.05$, is indicated by asterisks, and $p$ values are presented in parentheses): $76-90$ years $(p=0.016)$ and $>90$ years $(p=$ 0.0002 ), respectively

ducted studies, including our own, no telomere analysis of the human pituitary gland has yet been reported. In the present study, we investigated the dynamics of telomere (TRF) length in the human pituitary gland.

Sawabe et al. have reported age-associated changes in human organ weights based on 1,615 consecutive autopsies conducted on patients aged 60-109 years at our institution (Tokyo Metropolitan Geriatric Hospital) (2006). The investigated organs included the brain, heart, lung, liver, kidney, spleen, hypophysis (pituitary gland), thyroid, and adrenal gland. This valuable data source revealed that the pituitary had several notable characteristics from the viewpoint of aging. First, the gross size of the pituitary was generally better preserved during aging than any other organ and remained almost constant up to 80 years of age. Second, the pituitary gland demonstrated no age-related changes in terms of histology; even in extremely old individuals, hardly any age-related inflammatory cell infiltration or degeneration was evident, with only mild fibrosis. Third, the pituitary gland plays a pivotal physiological role in hormonal homeostasis via the hypothalamus-pituitary axis. These three features, and the relatively rapid organogenesis of the pituitary indicated by Ki-67 immunostaining (see Supplementary materials, Table S2, Fig. S1), appeared to suggest that this organ might have unique characteristics in terms of telomere status, i.e., (1) the initial TRF length (the value for neonatal pituitary) was $14.2 \pm 1.1 \mathrm{kbp}($ mean $\pm \mathrm{SD})$, being highest among those for other systemic organs, and (2) intrinsic telomere length is well conserved throughout life. In fact, the TRF length for the pituitary gland was one of the longest in several individuals whose systemic organs were analyzed (Fig. 1, see also Takubo et al. 2002).

Many studies of telomere shortening with age have focused on a variety of human systemic tissues (Ishii et al. 2006). Many different methods and different representative values have been used in those studies, and this has made it difficult to achieve an integrated understanding of the huge amount of archived data for TRF length. In this study, we have re-evaluated these differences based on our own data and tried to provide practical clues for utilizing such archived data. Our data suggested that the peak and median values of TRF could be compared under certain circumstances. Furthermore, although the median values of TRF were usually less than mean values due to the skewed distribution of TRF, our study also suggested that the median and mean values were also comparable at least in terms of the annual attrition rates derived from these two different representative values.

The reported annual telomere reduction rates for various cells and tissues were within 25-100 bp per year. Our present results show that the values for pituitary (21 bp) were equivalent to, or below, the lowest part of this range, and slightly larger than those for heart and brain (gray matter) (Takubo et al. 2002; Nakamura et al. 2007; see also legend of Fig. S7). This feature can be explained in terms of the cell proliferation dynamics for this organ: that is, marked proliferation occurs at the early fetal stage up to 20 weeks of gestation, and then suddenly stops almost completely at around 37 weeks. After birth, the pituitary slowly grows and matures, and thereafter is very well preserved throughout life, without only minimal age-related changes. On the other hand, Frenck et al. (1998) have reported rapid loss of telomere length in peripheral blood cells of young individuals and a much slower rate of attrition in older adults. A longitudinal study of baboon hematopoietic cells has further confirmed this phenomenon (Bearlocher et al. 2007). These data matched those that were theoretically expected and defined the phenomenological aspect of telomere length regulation, strongly suggesting that telomeres shorten in a cell proliferation-dependent manner. 
With regard to the in vivo telomere dynamics of postmitotic cells, we have previously reported the age-related telomere length dynamics in the brain, a typical organ composed mainly of post-mitotic cells. Telomere lengths decreased only slightly up to the 70-79-year age range, but then inversely increased in individuals of more advanced age. Thus, the 90-100-year age group possessed significantly longer telomeres than individuals in their seventies (Nakamura et al. 2007). The present study demonstrated a pattern of telomere length dynamics in the pituitary similar to that of the cerebrum.

Cawthon et al. (2003) reported that individuals with shorter telomeres in blood had a high mortality rate due to heart disease and infectious disease, and speculated that such individuals might have increased rates of mortality due to many of the diseases typically associated with aging. This was based upon the notion that telomere lengths in blood cells accord with those in other tissues (Friedrich et al. 2000; Saretzki and von Zglinicki 2002). Indeed, several lines of evidence suggest that telomere length is a characteristic feature of human individuals/cells (Takubo et al. 2002; Baird et al. 2003). The present study further confirmed that telomere lengths in individuals were distributed over a wide range in the very early stage of life. On the other hand, data demonstrating differential cell-specific telomere attrition have been accumulated mainly through the use of quantitative fluorescence in situ hybridization (QFISH), the attrition rate showing variability even within the hematopoietic lineage (Bearlocher et al. 2007; Aida et al. 2010). Although it is obvious that both initial telomere length and attrition rate are the most crucial factors for correct estimation of telomere status, such fundamental data remain largely undetermined. The telomere dynamics investigated in the present study could constitute useful basic data for studies of this issue, especially in the context of initial telomere length.

In a previous study, we examined telomere length dynamics (in the cerebrum) in relation to autopsy data for the sample donors. The clinical data indicated a decrease in the rate of cancer death in individuals in their eighties and nineties relative to that for individuals in their sixties, and a paucity of cancer death in individuals in their eighties bearing long telomeres. On the basis of the overall data, we hypothesized that longer telomeres protected the genome from instability (a major cause of carcinogenesis) and were associated with increased longevity (Nakamura et al. 2007). This was the first report to suggest a relationship between telomere length and longevity in the context of the incidence of cancer-related death (Kappei and Londoño-Vallejo 2007). In the present study, we again confirmed the relationship between age and cancer death rate in the cohort we analyzed. On the other hand, telomere attrition is thought to be accelerated (or decelerated) by various environmental or epigenetic factors, such as oxidative stress (von Zglinicki et al. 1995), life stress (Epel et al. 2004), or alcohol intake (Aida et al. 2011) in a tissue/cell-specific manner, and telomere shortening may, in turn, trigger replicative senescence or chromosomal instability, leading to age-related degeneration or tumorigenesis (Hastie et al. 1990; Vaziri et al. 1993; Nakamura et al. 2007). Therefore, it is possible that individuals with longer telomeres are more likely to have better tolerance to the degenerative effects of aging. Unfortunately, we did not investigate TRF lengths in cancer tissues in the present study and therefore we were unable to compare them with the TRF lengths for the pituitary in the same patient. Since telomere dynamics in the pituitary show the highest degree of conservation throughout life, our findings provide indispensable fundamental data for future studies of telomere dynamics in vivo, and for investigating a number of still intriguing issues. Our findings also lend support to the contention that longer telomeres may exert a stronger protective effect against genome instability or age-related diseases, and are correlated with longevity.

Acknowledgments We thank Sachiko Nishimura and Yoshihiro Fujita for valuable assistance, and Dr. David Douglas for language editing. This work was supported by Grants-in-Aid for Scientific Research, C18590354 (to K.N.), B18659104 (to K.T.), C17590325 (to N.I.-S.), and C18659116, 20590389 (to N.I.) from the Ministry of Education, Culture, Sports, Science and Technology of Japan.

Open Access This article is distributed under the terms of the Creative Commons Attribution Noncommercial License which permits any noncommercial use, distribution, and reproduction in any medium, provided the original author(s) and source are credited.

\section{References}

Aida J, Izumo T, Shimomura N, Nakamura K, Ishikawa N, Matsuura M, Poon SS, Fujiwara M, Sawabe M, Arai T, 
Takubo K (2010) Telomere lengths in the oral epithelia with and without carcinoma. Eur J Cancer 46:430-438

Aida J, Yokoyama A, Izumiyama N, Nakamura K, Ishikawa N, Poon SSS, Fujiwara M, Sawabe M, Matsuura M, Arai T, Takubo K (2011) Alcoholics show reduced telomere length in the oesophagus. J Pathol 223:410-416

Allsopp RC, Vaziri H, Patterson C, Goldstein S, Younglai EV, Futcher AB, Greider CW, Harley CB (1992) Telomere length predicts replicative capacity of human fibroblasts. Proc Natl Acad Sci USA 89:10114-10118

Baird DM, Rowson J, Wynford-Thomas D, Kipling D (2003) Extensive allelic variation and ultrashort telomeres in senescent human cells. Nat Genet 33:203-207

Bearlocher GM, Rice K, Vulto I, Lansdorp PM (2007) Longitudinal data on telomere length in leukocytes from newborn baboons support a marked drop in stem cell turnover around 1 year of age. Aging Cell 6:121-123

Blackburn EH (1991) Structure and function of telomeres. Nature 350:569-573

Cawthon RM, Smith KR, O'Brien E, Sivatchenko A, Kerber RA (2003) Association between telomere length in blood and mortality in people aged 60 years or older. Lancet 361:393-395

Epel ES, Blackburn EH, Lin J, Dhabhar FS, Adler NE, Morrow JD, Cawthon RM (2004) Accelerated telomere shortening in response to life stress. Proc Natl Acad Sci USA 101:17312-17315

Frenck RW Jr, Blackburn EH, Shannon KM (1998) The rate of telomere sequence loss in human leukocytes varies with age. Proc Natl Acad Sci USA 95:5607-5610

Friedrich U, Griese E-U, Schwab M, Fritz P, Thon K-P, Klotz U (2000) Telomere length in different tissues of elderly patients. Mech Ageing Dev 119:89-99

Grant JD, Broccoli D, Muquit M, Manion FJ, Tisdall J, Ochs MF (2001) Telometric: a tool providing simplified, reproducible measurements of telomeric DNA from constant field agarose gels. Biotechniques 31:1314-1316

Greider CW (1996) Telomere length regulation. Annu Rev Biochem 65:337-365

Harley CB, Futcher AB, Greider CW (1990) Telomeres shorten during ageing of human fibroblasts. Nature 345:458-460

Hastie ND, Dempster M, Dunlop MG, Thompson AM, Green DK, Allshire RC (1990) Telomere reduction in human colorectal carcinoma and with ageing. Nature 346:866-868

Ishii A, Nakamura K, Kishimoto H, Honma N, Aida J, Sawabe M, Arai T, Fujiwara M, Takeuchi F, Kato M, Oshimura M, Izumiyama N, Takubo K (2006) Telomere shortening with aging in the human pancreas. Exp Gerontol 41:882-886

Kappei D, Londoño-Vallejo A (2007) Telomere length inheritance and aging. Mech Ageing Dev 129:17-26

Levy MZ, Allsopp RC, Futcher AB, Greider CW, Harley CB (1992) Telomere end-replication problem and cell aging. J Mol Biol 225:951-960
Lopes MBS, Pernicone PJ, Scheithauer BW, Horvath E, Kovacs K (2007) Pituitary and sellar region. In: Mills SE (ed) Histology for pathologists, 3rd edn. Lippincott Williams \& Wilkins, Philadelphia, pp 321-344

Nakamura K, Izumiyama-Shimomura N, Sawabe M, Arai T, Aoyagi Y, Fujiwara M, Tsuchiya E, Kobayashi Y, Kato M, Oshimura M, Sasajima K, Nakachi K, Takubo K (2002) Comparative analysis of telomere lengths and erosion with age in human epidermis and lingual epithelium. J Invest Dermatol 119:1014-1019

Nakamura K, Takubo K, Izumiyama-Shimomura N, Sawabe M, Arai T, Kishimoto H, Fujiwara M, Kato M, Oshimura M, Ishii A, Ishikawa N (2007) Telomeric DNA length in cerebral gray and white matter is associated with longevity in individuals aged 70 years or older. Exp Gerontol 42:944-950

Olovnikov AM (1971) Principles of marginotomy in template synthesis of polynucleotides. Doklady Akad Nauk USSR 201:1496-1499

Olovnikov AM (1996) Telomeres, telomerase, and aging: origin of the theory. Exp Gerontol 31:443-448

Sawabe M, Saito M, Naka M, Kasahara I, Saito Y, Arai T, Hamamatsu A, Shirasawa T (2006) Standard organ weights among elderly Japanese who died in hospital, including 50 centenarians. Pathol Int 56:315-323

Saretzki G, von Zglinicki T (2002) Replicative aging, telomeres, and oxidative stress. Ann N Y Acad Sci 959:24-29

Schwartz DC, Cantor CR (1984) Separation of yeast chromosomesized DNAs by pulsed field gradient gel electrophoresis. Cell 37:67-75

Statistics and Information Department, Ministry’s Secretariat, Ministry of Health, Labour and Welfare Japan (ed) (2006) Statistical abstracts of health and welfare in Japan 2005. Health \& Statistics Association Japan

Takubo K, Nakamura K, Izumiyama N, Sawabe M, Arai T, Esaki Y, Tanaka Y, Mafune K, Fujiwara M, Kammori M, Sasajima K (1999) Telomere shortening with aging in human esophageal mucosa. Age 22:95-99

Takubo K, Izumiyama-Shimomura N, Honma N, Sawabe M, Arai T, Kato M, Oshimura M, Nakamura K (2002) Telomere lengths are characteristic in each human individual. Exp Gerontol 37:523-531

Vaziri H, Schachter F, Uchida I, Wei L, Zhu X, Effros R, Cohen D, Harley CB (1993) Loss of telomeric DNA during aging of normal and trisomy 21 human lymphocytes. Am J Hum Genet 52:661-667

Verdun RE, Karlseder J (2007) Replication and protection of telomeres. Nature 447:924-931

von Zglinicki T, Saretzki G, Döcke W, Lotze C (1995) Mild hyperoxia shortens telomeres and inhibits proliferation of fibroblasts: a model for senescence? Exp Cell Res 220:186-193

Watson J (1972) Origin of concatemeric T7 DNA. Nat N Biol 239:197-201 